\title{
Ciliados nas cavidades do estômago de bovinos
}

\author{
[Ciliates present in the stomach chambers of bovines] \\ G.M.M. Salvio ${ }^{1}$, M. D'Agosto ${ }^{2}$ \\ ${ }^{1}$ Rua Florinda Tepedino Laroca, 18, Vila Laroca \\ 36660-000 - Além Paraíba, MG \\ ${ }^{2}$ Instituto de Ciências Biológicas, Campus Universitário - UFJF \\ Juiz de Fora, MG
}

Recebido para publicação em 13 de julho de 2000.

Recebido para publicação, após modificações, em 23 de abril de 2001

E-mail: dagosto@icb.ufjf.br

\section{RESUMO}

O presente trabalho teve como objetivo identificar e quantificar os gêneros de ciliados do rúmen, retículo, omaso e abomaso de bovinos abatidos em matadouro municipal. Foram coletados $45 \mathrm{ml}$ de conteúdo de cada cavidade de 30 animais. No momento da coleta foram medidos temperatura e pH e as amostras posteriormente analisadas em laboratório. Os resultados mostraram que o ambiente do rúmen e do retículo é bastante estável com temperatura média entre 36 e $37^{\circ} \mathrm{C} \mathrm{e} \mathrm{pH} \mathrm{6,5.} \mathrm{Os} \mathrm{gêneros} \mathrm{mais} \mathrm{encontrados}$ no rúmen foram Entodinium (65,0\%), Isotricha (7,0\%), Diplodinium (5,7\%), Ostracodinium (5,1\%), Eremoplastron (4,5\%), Dasytricha (3,8\%) e outros sete gêneros que totalizaram os $8,7 \%$ restantes. No retículo os resultados foram Entodinium (35,2\%), Isotricha (23,4\%), Dasytricha (15,4\%) Eodinium (7,2\%), Eremoplastron (5,0\%), Diplodinium (4,3\%), Ostracodinium (3,8\%) e outros cinco gêneros totalizando os 5,6\% restantes. No omaso foram encontrados os mesmos gêneros, porém em menor quantidade que no rúmen e retículo. No abomaso não foram encontrados ciliados. A análise do perfil populacional das amostras do rúmen mostrou predominância do tipo 0 (87\%), seguido do tipo B (13\%).

Palavras-chave: Bovino, ciliado, cavidade gástrica

\begin{abstract}
The objective of this study was to collect and to quantify the ciliate genera present in the rumen, reticulum, omasum and abomasum in 30 slaughtered bovines. Rumen, reticulum, omasum and abomasum were sampled and their contents $(45 \mathrm{ml})$ were collected and evaluated for temperature, $\mathrm{pH}$ and ciliates population. The results showed that the rumen and reticulum environments are quite stable with average temperature between 36 and $37^{\circ} \mathrm{C}$ and $\mathrm{pH}$ 6.5. The ciliate population in the rumen was Entodinium (65.0\%), Isotricha (7.0\%), Diplodinium (5.7\%), Ostracodinium (5.1\%), Eremoplastron (4.5\%), Dasytricha (3.8\%) and seven other genera which made $8.7 \%$. In the reticulum, the population was Entodinium (35.2\%), Isotricha (23.4\%), Dasytricha (15.4\%) Eodinium (7.2\%), Eremoplastron (5.0\%), Diplodinium (4.3\%), Ostracodinium (3.8\%) plus $5.6 \%$ of other five genera. The same genera were found in the omasum but in a smaller percentage than in the rumen and reticulum. No ciliates were found in the abomasum. The population profile analysis of the rumen samples has showed the predominance of type $O$ (87\%) followed by type B (13\%).
\end{abstract}

Keywords: Cattle, ciliate, gastric cavities 


\section{INTRODUÇÃO}

A participação de microrganismos na digestão de alimentos de ruminantes permite maior aproveitamento de carboidratos presentes na celulose. Como os ruminantes não produzem enzimas que as digerem, eles desenvolveram uma relação simbiótica com microrganismos capazes de digerirem a celulose (Dehority, 1985). A maior vantagem dos ruminantes é assimilar pelo menos parte da energia contida em dietas ricas em celulose e hemicelulose. Além disso, há outras vantagens, amônia e uréia podem ser convertidas em proteínas por meio de relações simbióticas com microrganismos. Qualquer amônia ou uréia ingerida junto com o alimento ou produzida pelos microrganismos pode ser convertida em proteína que pode ser digerida e absorvida pelo hospedeiro (Withers, 1992). Da microbiota ruminal fazem parte protistas ciliados, bactérias, fungos e protistas flagelados. A microbiologia do rúmen é complexa devido ao grande número de organismos presentes, suas diferentes natureza e mudança de população, que resultam da mudança da dieta do ruminante (Teixeira, 1992).

Os primeiros protozoários de rúmen foram observados por Gruby \& Delafond (1843). A quantidade e as espécies de protistas não são necessariamente as mesmas dentro da mesma espécie de ruminante, apesar de alguns ciliados de rúmen serem específicos a determinados hospedeiros. A variação pode depender do tipo de alimentação, da distribuição geográfica e do antagonismo entre certas espécies (Abou-Akkada et al., 1968).

O papel dos ciliados de rúmen ainda não foi bem esclarecido. Vários trabalhos (Jouany, 1990; Williams \& Coleman, 1991; Williams et al., 1993) apontam fatores positivos e negativos da presença desses organismos em ruminantes. Williams \& Coleman (1991) apresentaram resultados de vários autores sobre a influência da presença ou ausência de ciliados no conteúdo ruminal no desenvolvimento e produtividade dos hospedeiros.

O presente trabalho teve como objetivos realizar levantamento de ciliados que ocorrem em bovinos abatidos no município de Além Paraíba, MG, quantificar os ciliados encontrados no rúmen, retículo, omaso e abomaso de bovinos, e avaliar a relação entre esses gêneros e os fatores que influenciam o perfil populacional encontrado.

\section{MATERIAL E MÉTODOS}

O trabalho foi desenvolvido no município de Além Paraíba, Minas Gerais, e a análise do material, identificação e quantificação dos ciliados foram feitas na Universidade Federal de Juiz de Fora, em laboratório do Curso de Mestrado em Ciências Biológicas - Comportamento e Ecologia Animal.

A região onde se localiza o município de Além Paraíba é de clima tropical, com duas estações definidas, a estação seca, de abril a setembro, e a estação chuvosa, de outubro a março.

Foram obtidos conteúdo de rúmen, retículo, omaso e abomaso de 30 animais procedentes de fazendas de Além Paraíba e recém-abatidos no Matadouro Municipal, totalizando 120 amostras, 30 de cada compartimento. No momento de obtenção das amostras foram coletadas informações sobre os hospedeiros e locais de procedência. Os animais foram escolhidos aleatoriamente sendo todos mestiços resultantes do cruzamento de bovinos da raça Gir e Holandesa, de ambos os sexos. Apresentavam idade entre quatro e cinco anos e peso médio de $225 \mathrm{~kg}$. Os animais foram alimentados nas fazendas de origem apenas com capim-braquiária, capim-gordura e/ou capim-colonião. As coletas foram realizadas nos dias 22 de julho e 5 de agosto de 1998, após as 18 horas, momento de abate no Matadouro Municipal.

Os animais chegaram ao matadouro pelo menos 12 horas antes do abate, e não receberam nenhum tipo de alimento durante esse período, exceto água à vontade. As amostras foram obtidas diretamente das cavidades após o abate e imediatamente fixadas com formalina a 18,5\% e conservadas em frascos devidamente identificados com o número do animal e a cavidade de onde foi retirado o conteúdo. 
As avaliações quantitativas e qualitativas se basearam em Dehority (1984) e de acordo com a modificação proposta por D'Agosto \& Carneiro (1999), na qual o corante verde brilhante foi substituído por solução de lugol. A identificação dos gêneros de ciliados encontrados no rúmen e no retículo foi baseada em Ogimoto \& Imai (1981). No momento da coleta das amostras foram medidos pH e temperatura de cada compartimento, utilizando-se pHmetro Hanna modelo HI8314.

As análises do perfil populacional registrado no rúmen e no retículo tiveram como princípio básico as observações propostas por Eadie (1962a,b).

Fez-se análise de variância pelo General Linear Model e comparação entre médias aplicando-se o teste de Tukey $(\mathrm{P}<0,05)$.

\section{RESULTADOS E DISCUSSÃO}

A maior quantidade de protozoários ciliados foi encontrada no rúmen (Tab. 1), predominando representantes da família Ophryoscolecidae, principalmente do gênero Entodinium (Tab. 2).

Tabela 1.Quantidade de ciliados por ml de conteúdo, por família, encontrados nas quatro cavidades do estômago de bovinos recém-abatidos em Além Paraíba, MG

\begin{tabular}{lrrrc}
\hline Família & Rúmen & Retículo & Omaso & Abomaso \\
\hline Blepharocorythidae & 32000 & 11200 & 0 & 0 \\
Isotrichidae & 628800 & 1235200 & 4800 & 0 \\
Ophryoscolecidae & 5322200 & 1952000 & 14400 & 0 \\
Total & 5983000 & 3198400 & 19200 & 0 \\
\hline
\end{tabular}

Tabela 2. Número médio e percentual de ciliados encontrados por ml de conteúdo das quatro cavidades do estômago de bovinos recém-abatidos no Matadouro Municipal de Além Paraíba, MG

\begin{tabular}{|c|c|c|c|c|c|c|c|c|c|c|}
\hline \multirow{2}{*}{ Gênero } & \multicolumn{2}{|c|}{ Rúmen } & \multicolumn{2}{|c|}{ Retículo } & \multicolumn{2}{|c|}{ Omaso } & \multicolumn{2}{|c|}{ Abomaso } & \multicolumn{2}{|c|}{ Total } \\
\hline & $\bar{X}$ & $\%$ & $\bar{X}$ & $\%$ & $\bar{X}$ & $\%$ & $\bar{X}$ & $\%$ & $\bar{X}$ & $\%$ \\
\hline Isotricha & 404800 & 6,9 & 744000 & 23,4 & 3200 & 9,5 & - & - & 1152000 & 12,7 \\
\hline Dasytricha & 224000 & 3,8 & 488000 & 15,3 & 8000 & 23,8 & - & - & 720000 & 8,0 \\
\hline Charonina & 32000 & 0,5 & 11200 & 0,3 & - & - & - & - & 43200 & 0,5 \\
\hline Entodinium & 3782400 & 64,9 & 1120000 & 35,2 & 11200 & 33,3 & 6400 & 100 & 6060800 & 54,5 \\
\hline Eudiplodinium & 129600 & 2,2 & 48000 & 1,5 & 1600 & 4,7 & - & - & 179200 & 2,0 \\
\hline Eremoplastron & 264000 & 4,5 & 160000 & 5,0 & 1600 & 4,7 & - & - & 425600 & 4,7 \\
\hline Ostracodinium & 299200 & 5,1 & 120000 & 3,7 & 1600 & 4,7 & - & - & 420800 & 4,6 \\
\hline Eodinium & 150400 & 2,6 & 228800 & 7,2 & 1600 & 4,7 & - & - & 380800 & 4,2 \\
\hline Polyplastron & 1600 & 0,02 & -- & & - & - & - & - & 1600 & 0,01 \\
\hline Diplodinium & 334400 & 5,7 & 137600 & 4,3 & - & - & - & - & 472000 & 5,2 \\
\hline Diploplastron & 9600 & 0,2 & 8000 & 0,2 & - & - & - & - & 17600 & 0,2 \\
\hline Metadinium & 59200 & 1,0 & 38400 & 1,2 & 1600 & 4,7 & - & - & 99200 & 1,1 \\
\hline Epidinium & 128000 & 2,2 & 73600 & 2,3 & 3200 & 9,5 & - & - & 204800 & 2,3 \\
\hline Total & 5899200 & 65,3 & 3177600 & 35,1 & 33600 & 0,37 & 6400 & 0,07 & 9036800 & 100 \\
\hline
\end{tabular}

No retículo há nitidamente maior número de protozoários representantes da família Isotrichidae em relação ao encontrado no rúmen, porém representantes do gênero Entodinium foram os protistas mais freqüentes nessa cavidade.

No omaso, a freqüência de ciliados diminuiu acentuadamente em relação às cavidades anteriores (Tab. 1), contudo os gêneros encontrados foram semelhantes. No abomaso, que representa o estômago químico onde são produzidos a pepsina e o ácido clorídrico responsáveis pela digestão química nos ruminantes, não foram encontrados ciliados. Certamente o pH ácido inviabilizou o desenvolvimento de 
microrganismos, pois sabe-se que os protozoários são inibidos em $\mathrm{pH}$ inferior a 5,5 e morrem quando o pH é 4,5 (Ruiz, 1992).

Considerando-se as quatro cavidades, observou-se o gênero Entodinium como predominante, seguido de Isotricha, Dasytricha, Diplodinium, Eremoplastron, Ostracodinium, Eodinium, Epidinium, Eudiplodinium, Metadinium, Charonina, Diploplastron e Polyplastron.

Ciliados do gênero Entodinium são predominantes no rúmen (Towne et al., 1990; Willians \& Coleman, 1991; Franzolin et al., 1997), podendo chegar a compor 95\% do total de ciliados do rúmen (D'Agosto et al., 1998). Essa predominância foi assinalada em todos os compartimentos do estômago onde foram encontrados ciliados.

Os dados de temperatura e $\mathrm{pH}$ demonstraram que rúmen, retículo, omaso e abomaso representam ecossistemas constantes. Estas observações concordam com as afirmações de Teixeira (1992) para o sistema ruminal, podendo ser ampliadas para outros compartimentos do estômago. Considerando as medições realizadas imediatamente após as coletas nos interiores de cada cavidade, o rúmen apresentou temperatura média de $37,4^{\circ} \mathrm{C}$ e $\mathrm{pH}$ médio de 6,5 . Estes valores de temperatura e $\mathrm{pH}$ do rúmen estão dentro da faixa de variação apresentada por Dehority (1987) considerada como normal. Os valores de temperatura e $\mathrm{pH}$ dos demais compartimentos não foram relatados por outros autores. No retículo a temperatura média foi de $36,7^{\circ} \mathrm{C}$ e o $\mathrm{pH}$ médio de 6,5 . No omaso foram $36,5^{\circ} \mathrm{C}$ e 5,3 , e no abomaso $37,1^{\circ} \mathrm{C}$ e 3,8 . Nesse último compartimento o baixo $\mathrm{pH}$ é reflexo da presença do ácido clorídrico produzido.

Rúmen e retículo apresentaram condições de temperatura e $\mathrm{pH}$ bastante semelhantes. O mesmo pode-se dizer quanto aos gêneros de protozoários ciliados encontrados em ambas as cavidades. Variou apenas a ocorrência de determinados gêneros no rúmen e no retículo. O gênero Entodinium foi o predominante nas duas cavidades, contudo nota-se diminuição na porcentagem desses ciliados no retículo e maior número de representantes da família Isotrichidae, como Dasytricha e Isotricha. Essa variação é atribuída ao comportamento de escape do rúmen ao retículo de isotriquídeos hora após a alimentação dos bovinos (Salvio \& D'Agosto, 1999).

O omaso, caracterizado por Towne \& Nagajara (1990) como um nicho ecológico capaz de sustentar o desenvolvimento de microorganismos, apresentou valores abaixo dos encontrados no rúmen e retículo. Os gêneros são semelhantes, excetuando-se o fato de não terem sido encontrados Charonina, Polyplastron, Diplodinium e Diploplastron.

Tanto no rúmen quanto no retículo população do tipo $\mathrm{K}$, caracterizada pela presença do gênero Elytroplastron (Willians \& Coleman, 1991) não foi registrada. Em 87\% dos animais a população foi do tipo $\mathrm{O}$, caracterizada pela predominância de Entodinium e isotriquídeos e pela ausência de grandes entodinomorfidas (Willians \& Coleman, 1991). Não foi encontrada população do tipo A, caracterizada pela presença do gênero Polyplastron e usualmente acompanhado pelo gênero Diploplastron (Eadie, 1962a). Quatorze bovinos apresentaram população do tipo B no rúmen, caracterizada pela presença dos gêneros Eudiplodinium e Epidinium (Eadie, 1962a). Desses, 12 apresentavam ambos os gêneros e foram registrados em dois animais o gênero Eudiplodinium não associado a Epidinium.

No retículo, 13 animais apresentaram população do tipo B. Em quatro deles registrou-se presença de Eudiplodinium não associada à de Epidinium e em outros três animais foram encontrados Epidinium não associado a Eudiplodinium.

Ainda não é suficientemente claro o papel dos ciliados no rúmen. Isso dificulta estabelecer um conceito para a relação entre ciliados e ruminantes. Parece mais conveniente considerar o rúmen como um ecossistema fechado, onde protozoários e bactérias mantêm uma relação próxima, que em conjunto afeta positiva ou negativamente a digestão e, conseqüentemente, o desempenho produtivo dos ruminantes. 


\section{REFERÊNCIAS BIBLIOGRÁFICAS}

ABOU AKKADA, A.R., BARTLEY, E.E., FINA, L.R. Ciliate protozoa in the rumen of the lactating cow. J. Dairy Sci., v.52, p.1088-1091, 1968.

D’AGOSTO, M., SANTA-ROSA, M.R., AROEIRA, L.J.M. et al. Influência da dieta no comportamento da população de ciliados do rúmen. Arq. Bras. Med. Vet. Zootec., v.50, p.153-159, 1998.

D'AGOSTO, M., CARNEIRO, M.E. Evaluation of lugol solution used for counting rumen ciliates. Rev. Bras. Zool., v.16, p.725-729, 1999.

DEHORITY, B. A. Protozoa of the digestive tract of herbivorous mammals. Insect Sci. Applic., v.7, p. 279-296, 1985.

DEHORITY, B.A. Evaluation of subsampling and fixation procedures used for counting rumen protozoa. Appl. Environ. Microbiol., v.48, p. 182-185, 1984.

DEHORITY, B.A. Rumen microbiology. The Ohio State University, 1987. 125p.

EADIE, M. Inter-relationships between certain rumen ciliate protozoa. J. Gen. Microbiol., v.29, p.579-588, 1962 b.

EADIE, M. The development of rumen populations in lambs and calves under various conditions of management. $J$. Gen. Microbiol., v.29, p. 563-578, 1962a.

FRANZOLIN, R., FRANZOLIN, M.H.T., NOGUEIRA-FILHO, J.C.M. et al. Avaliação comparativa da fauna ruminal e da degradabilidade entre búfalos e bovinos alimentados à base de cana-de-açúcar. In: Reunião Anual da Sociedade Brasileira de Zootecnia, 24, Juiz de Fora, 1997. Anais... Juiz de Fora: SBZ, 1997.

GRUBY, D., DELAFOND, O. Recherches sur des animalcules se développant en grand nombre dans l'estomac et dans les intestins, pendant la digestion des animaux herbivores et carnivores. Compt. Rend. Acad. Sci., v.17, p.1304-1308, 1843.

JOUANY, J.P. Defaunation of the rumen. Indian Summer Course on Rumen Microbial Metabolism and Ruminant Digestion, vol. 2. France, Clermon-Ferrand/Theix, 1990.

OGIMOTO, K., IMAI, S. Atlhas of rumen microbiology. Tokyo: Japan Scientific Societies Press, viii+231 p.1981.

RUIZ, R. L. Microbiologia zootécnica. São Paulo: Ed. Roca, 1992. 314p.

SALVIO, G.M.M., D'AGOSTO, M. Avaliação do comportamento de escape e migração de ciliados isotriquídeos no sistema rúmen-retículo de bovinos. Rev. Bras. Zoocienc., v.1, p.41-52, 1999.

TEIXEIRA, J. C. Nutrição de ruminantes. Lavras: Edições FAEPE, XVIII+239p, 1992.

TOWNE, G., NAGAJARA, T.G. Omasal ciliated protozoa in cattle, bison and sheep. Appl. Environ. Microbiol., v.56, p.409-412, 1990.

TOWNE, G., NAGAJARA, T.G., BRANDT, R.T. et al. Ruminal ciliated protozoa in cattle fed finishing diets with or without suplemental fat. J. Anim. Sci., v.68, p.2150-2155, 1990.

WHITERS, P. C. Comparative animal physiology. New York: Saunders College Publishing, 1992. 949p.

WILLIANS, A.G., COLEMAN, G.S. The rumen protozoa. Springer-Verlag, New York Inc., 1991. 423p.

WILLIANS, A.G., JOBLIN, K.N., BUTLER, R.D. et al. Interactions bactéries-protiste dans le rumen. Ann. Biol., v.32, p.14-29, 1993. 